\title{
The Globalization of Chinese Online Literature via a Bourdieusian Capital Perspective A Case Study of Coiling Dragon
}

\author{
Jialing $\mathrm{Wu}^{1}$ Chunfang $\mathrm{Yi}^{1, *}$ \\ ${ }^{1}$ School of Foreign Studies, Northwestern Polytechnical University, Xi'an, Shaanxi 710000, China \\ *Corresponding author. Email: yi_chunfang@nwpu.edu.cn
}

\begin{abstract}
The globalization of Chinese online literature has become more and more phenomenal in recent years. Based on the perspectives of translation sociology and particularly of the French sociologist Bourdieu's capital theory, which involves economic capital, cultural capital, symbolic capital and social capital, this article analyzes the process of globalization of one of the earliest overseas-published Chinese online literature Coiling Dragon, in order to explain the success of it in terms of capital flow in the transcultural dissemination of translated Chinese texts. With the sociological analysis of the translated Coiling Dragon, it is concluded that the successful globalization of Coiling Dragon is closely related to the author, the translator, the publishing agency, and the governmental policy. These factors, together with the play of economic capital, cultural capital, symbolic capital and social capital, establish Coiling Dragon's ranking at the top five in the fantasy section of Wuxiaworld, and is evaluated as 'overwhelmingly recommended' among overseas readers. This article tries to map out the dynamic relationships among these factors and demonstrate that the cultural capital and symbolic capital the original author holds and the cultural, economic and social capital the translator holds help Coiling Dragon successfully go global.
\end{abstract}

Keywords: Coiling Dragon, Pierre Bourdieu, Capital theory, Cultural capital, Translation sociology, "Going global".

\section{INTRODUCTION}

The overseas dissemination of Chinese popular literature via networks has been increasing and drawn academic attention. According to Chinese Online Literature Going to Sea in 2020, the overseas market of Chinese Internet literature reached 460 million yuan (iResearch, 2020). [1] At the same time, iResearch (2020) has noted that the number of online literary works exported overseas has exceeded 10,000 by 2019 , and translated online literary works reached 3, 452 in 2019. [1] With the guidance of national policies and the promotion of corporate establishments, the scale of overseas Chinese online literature will be further expanded in the future.

At present, the main research focus in the field of overseas communication of Chinese online literature are as follows: 1. Current Situations of
Chinese Online Literature Overseas. According to Alexa statistics on March 1, 2019, Wuxiaworld, the largest Chinese online literature translation website in the English world, has an average daily visitors of approximately 672,000 and an average daily page visit of approximately 6, 585, 600 (Xu \& Hua, 2020). [2] The overseas market has great potential for development, but it still faces many problems, such as the lack of systematization and standardization in the development of the translation industry overseas, and the increasing infringement of intellectual property rights for online literary works. 2. Research on the Translation Strategy of Chinese Network Literature. Yuan (2021) took the fantasy novel Coiling Dragon as an example to analyze strategies of domestication and foreignization in the translation of Chinese online literature. [3] Ren (2020) focused on exploring new models for the translation of online martial arts novels. [4] 3. Research on 
Globalization Strategy of Chinese network literature. The spread of online literature is inseparable from the operation of promotional platforms, such as Wuxiaworld, Gravity, Start International, etc. Lv Zhenyan (2020) has provided many suggestions for the globalization strategies of Chinese online literature from a cross-cultural perspective, such as the protection of online literature's copyright, training professional translators through multiple channels, and establishing a screening mechanism for original online literature. [5]

Pan Long, or Coiling Dragon, is a work of Chinese online literature written by a popular Chinese (fantasy/kung fu) writer, known online as "I Eat Tomatoes (我吃西红柿)". This novel has been fully translated by Ren Wo Xing (RWX), and published in Wuxiaworld, causing a sensation abroad. Through the statistics from Wuxiaworld, the total number of comments on Coiling Dragon has reached 80,000 , of which there are more than 9,000 long comments (more than three lines of comments), and as many as 632 comments are generated by only counting the final chapter. Lin (2018) pointed out that for many overseas readers, Coiling Dragon is their first Chinese online novel. [6] The author believes that Coiling Dragon, as one of the first works to go overseas successfully, is of great significance to the exploration of successful path for the dissemination of many other Chinese network literature works.

Successful translation and dissemination is closely related to the sociological knowledge involved in the process. As an important theory in the field of sociology, Bourdieu's capital theory has played a great role in combining cultural capital, symbolic capital, and economic capital into the analysis of the circulation of cultural products. By adopting Bourdieu's capital theory to analyze the translation and dissemination of Coiling Dragon, it is expected that some experience and lessons can be obtained for guidance to the globalization of Chinese online literature. Therefore, this article analyzes the translation and dissemination process of Coiling Dragon based on Bourdieu's capital theory, showing how Pan Long, as a cultural product, has turned into cultural capital in the translation and literary field through the author, translator, publishing platform and promotional platform, and the construction and circulation of Coiling Dragon as cultural capital. Also, this paper demonstrates the interaction between the translator and the author, the publishing agency, and the relevant state departments in the translation field, as well as the important role the economic capital, social capital, and cultural capital have played in the successful dissemination of Coiling Dragon. The author hopes that this article can provide some reference for Chinese online literature and Chinese culture to go abroad.

\section{BOURDIEUSIAN THEORY}

Pierre Bourdieu is a famous French sociologist and psychologist. In Bourdieu's Cultural Production Theory, he proposed a famous analysis model: [(habitus) (capital)] + field $=$ practice. [7] Bourdieu (1984) believes that practice is the result of the interaction between field, habitus and capital. [8] Translation activities are a type of social practice, so according to his analytical mode, translators fight in the field of power, with all kinds of habitus and capital, thus the field of translation is formed.

\subsection{Field}

Bourdieu (1997) defined the field as follows: In analytic terms, a field may be defined as a network, or a configuration, of objective relations between positions. These positions are objectively defined, in their existence and in the determinations they impose upon their occupants [...] as well as by their objective relation to other positions. [9]

Human society is composed of countless different fields, such as the literary field, the economic field, the social field, the translation field, etc. Each field has different rules and different types of participants. For example, the translation field is mainly composed of authors, translators, publishers, readers and so on. With the development and popularity of online literature going overseas, many foreign translations are not only published by traditional foreign publishing houses, but also on famous international websites such as Wuxiaworld, Gravity Tales, etc.

The translation and dissemination of Coiling Dragon is conducted in a field whose main participants include "I eat tomatoes", who was the original author of Coiling Dragon, the translator RWX, the relevant online literature publishing platform - Wuxiaworld, and related promotional platforms. In this field, different participants conflict with each other, creating and accumulating habitus and capital in the process. 


\subsection{Habitus}

Bourdieu was the first to use Habitus to discuss social issues. Habitus is a concept that guides our ways of constructing objects of study, highlighting issues of significance and providing a means of thinking relationally about those issues (Bourdieu, 1993). [10] It focuses on our ways of acting, feeling, thinking and being. In order to better understand and define it, he put forward the concept of disposition. Disposition has two core elements, one refers to "the dialectic of the internalization of externality and the externalization of internality" (Bourdieu, 1977); the other refers to a habitual state, such as hobbies, tendencies, etc. [11] Habitus is the internalization of field rules. Whoever has the habituation that is more in line with the rules of the field is more likely to win and get power in the field. At the same time, habituation can be continuously adjusted and adapted to changes in the field, and will also change due to some political and economic influences.

In the field where Coiling Dragon is located, the author "I eat tomatoes" and the translator RWX show distinctive habitus. "I eat tomatoes" loves reading and writing, and his main preference is martial arts novels with exciting plots. These habits influenced his own literary creation, and eventually made Coiling Dragon a fantasy martial arts novel that wins with the quality of the plot. The translator RWX, as a Chinese descent living in America, has been influenced by Chinese culture since he was a child. He also likes to read fantasy and online martial arts novels, which makes him interested in reading Coiling Dragon and finally translating it.

\subsection{Capital}

Bourdieu (1984) believes that there are four forms of capital, namely economic capital, cultural capital, social capital, and symbolic capital. [8] Symbolic capital is transformed from economic, cultural, and social capital. Economic capital can be converted into money. Cultural capital is mainly related to education and knowledge, and can be converted into economic capital. Social capital consists of social relationships, including social connections and promotional resources. Symbolic capital refers to someone's status, and the role in a certain field. In the translation activity, the translator will evaluate various forms of capital he possesses, and then use the capital rationally, and finally present it in the translation strategy and the dissemination of it. To some extent, the choice of translation strategy is the result of the translator's comprehensive consideration of various capitals. Whether the translation results are widely recognized is a manifestation of the power of capital deployment.

The original author "I Eat Tomatoes" is the "platinum god (baijin)" of the Chinese website Qi Dian. Evaluative ranking on the author's popularity at Qi Dian has five levels, the highest of which is platinum god, which means that almost every novel of the author is of super high popularity. "I Eat Tomatoes" won the title of "King of Internet Literature" in 2018 and was selected as one of the 100 industry figures in Internet literature field for the past 20 years in 2020 . Therefore, he has a high reputation in the field of Internet literature, that is, he has a relatively high reputation as well as a high symbolic capital in the field of Internet literature.

\section{ANALYSIS OF THE TRANSLATION PROCESS OF COILING DRAGON BASED ON CAPITAL THEORY}

The successful translation of Pan Long is the first step also the most important step in its globalization strategy. According to Chen Yunuo (2013), the translation is regarded as an essential medium for the circulation, transmission, and accumulation of cultural capital. [12] Meanwhile, translation is also the bearer and manifestation of cultural capital and is responsible for cultural exchange between different nations. Coiling Dragon, as a new cultural product, has gone to global since the interaction between the author, the translator, the publishing platform and the promotional form in the translation field. The globalization process of it also can be seen as the circulation, transmission and accumulation of cultural capital, with the help of other capital such as symbol capital and economic capital.

The network literature translation field mainly includes authors, translators, platforms, etc. The globalization process of Coiling Dragon is divided into the following steps: First, the original author of Pan Long is the platinum god, and Pan Long in 2008 has achieved great results: it has recorded 94 million views and 7.7 million reader recommendations. This symbolic capital from the author and Pan Long has attracted the attention of the translator RWX. In addition, because the author holds rich cultural capital, Pan Long has a relatively high quality, which strengthens RWX's determination in translating the book. The translator 
successfully completed the translation work relying on his cultural capital of reading Chinese martial arts novels from an early age and translating martial arts novels for many years. After that, he relied on interpersonal relationships to obtain more social capital. At the same time, his own website Wuxiaworld also provided a good chance for him to publish novels and increase reading volume by social capital and promotional resources; finally, our country's promotion to Coiling Dragon under the national policy of "Chinese Internet Literature Going to Sea" also helped him achieve success. Therefore, the following will be based on Bourdieu's capital theory and try to analyze the globalization process of Coiling Dragon from the perspective of text selection, translation production and translation dissemination.

\subsection{The Capital Accumulation of the Original Author and His Writing of Pan Long}

The cultural capital accumulation about the original author "I eat tomatoes" lays a good foundation for RWX choosing to translate Pang Long. "I eat tomatoes", originally names Zhu Hongzhi, was born in Baoying, Jiangsu, and graduated from the Department of Mathematics, Soochow University in 2009. He has liked to read martial arts novels since he was a child, especially in Jin Yong, Wolongsheng, and Gu Long. When he was in high school, he tried to write novels on Qidian Chinese website, and his first work Legend of Star Peak attracted considerable attention. When he was in college, his monthly income has been over 10,000 yuan after three months of writing. He basically achieves economic freedom after six months. He has written more than a dozen works on the Qi Dian website, such as Star Change, Coiling Dragon, Jiu Ding Ji, Swallowing the Starry Sky, and Reckless Desolation. Because he likes Jin Yong and $\mathrm{Gu}$ Long, who are very famous writers in China, many of his characters have the temperament that often appears in the novels of Jin Yong and $\mathrm{Gu}$ Long, such as strong, generous, unrestrained, etc. His experience in writing novels since high school also fosters the accumulation of his cultural capital.

Pan Long also becomes cultural capital, for "I eat tomatoes" has given the novel a high quality and popularity in China. On the domestic WeChat reading software platform, it is shown that 119,000 people are reading this book, and more than 8,080 people have commented on it. It has received "great work" with $87.4 \%$ of the comments. On the Douban platform, more than 5000 people have evaluated this, and its average score reached 7.6/10, which lays a good foundation for the translator to read and translate it.

The symbolic capital accumulation of the writer is also an important reason for Pan Long being translated. Because "I eat tomatoes" has written so many famous books, he was awarded the title of Top Ten Fairy Writers of Internet Literature for 20 Years, One Hundred Powerful God Writers, and One of the Hundred Industry Figures. Besides, he has won the title of "The King of Online Literature" at the 3rd Orange Melon Network Literature Award. Therefore, his fame arouses more people's attention to his works, including the translator RWX, who finally chose to translate one of his most popular work Pan Long.

Because of his passion for martial arts novels and Chinese online novels, his experience of writing novels since high school, his achievement and status in the Chinese online literature circle, he has accumulated cultural capital and symbolic capital, which has laid a certain degree of foundation for RWX to translate it. In addition, Pan Long as a famous cultural product and as cultural capital since its popularity in Chinese online literature market is also helpful for the translator to make the final decision. In conclusion, the capital accumulation of the original author and Pan Long is a prerequisite for the success of the globalization of Coiling Dragon.

\subsection{The Capital Accumulation of the Translator and the Translation Process of Coiling Dragon}

The cultural capital accumulation about the translator is of great importance for the production and globalization of Coiling Dragon. Ren Woxing, the translator of Coiling Dragon, formerly known as Lai Jingping, is a Chinese American. He moved to the United States with his parents at the age of three and has been growing up in the United States ever since. He has been familiar with American culture and mastered authentic English since he was a child. Therefore, he has accumulated the initial cultural capital. The American thinking mode also profoundly affected his translation strategies and skills, making it easier for foreign readers to accept online literary works from China. Because his parents are Chinese, he had access to Chinese literature. In order to read his favorite Chinese martial arts novels smoothly and better understand 
Chinese culture, he studied Chinese for several years in high school and university. He also worked with other martial arts enthusiasts on the Specnet forum, spending six or seven years translating almost all of Jin Yong and Gu Long's novels (Li \& Yang, 2018). [13] All the experiences further accumulated his cultural capital. Before Coiling Dragon, he has translated Jin Yong's Demi-Gods and Semi-Devils, Gu Long's The Magic Blade, etc., and improved translation skills, which further accumulated his cultural capital.

Also, his social capital accumulation has played a big role in promoting Coiling Dragon. For example, he met many like-minded friends in the translation circle when he translated some work on the Specnet forum, and gained initial social capital. Later, he came into contact with online literature. Because he liked Coiling Dragon such a work with easy-to-understand language and exciting plots, he started translating this work. While he was translating, he published the translated content on the Specnet forum. In the process of it, the English version of Coiling Dragon has gained the love of a large number of foreign netizens, with hundreds of thousands of hits a day in the Specnet forum (Li \& Yang, 2018). [13] In order to meet more people's reading needs, he established the website Wuxiaworld on December 22, 2014, and put Coiling Dragon on it for a long-term serialization. The platform also serves as a promotional medium and social capital, further fosters the successful dissemination of Coiling Dragon. After the publication of it, RWX began to try to enter the commercial field from the field of translation. In order to better improve the website, he added a forum area, a message area, a reward system, and introduced more excellent Chinese online novels into Wuxiaworld. The website has gradually become same famous as Webnovel, Gravity, etc., which attracted more readers to enter the "world of martial arts". As one of the most classic and exciting work, Coiling Dragon naturally got a lot of attention. After several years of growth, Wuxiaworld has become the world's largest Chinese-English translation platform. According to RWX, there are 300 to 350,000 unique user visits every day, with a total of nearly 3 billion clicks. Take the most popular novel as an example. It is updated twice a day, one chapter each time, and more than 200,000 people pay attention every day (Li \& Yang, 2018). [13] The state of development of the martial arts world, as a kind of social capital, continues to inject new vitality into the spread of Coiling Dragon. In addition, our country has put forward the strategy about "Chinese Online Literature Going Global" at the time. And domestic media made many reports on Coiling Dragon.

\subsection{Analysis of the Overseas Acceptance of Coiling Dragon}

The translation effect refers to whether the translated work has achieved the effect of cultural communication, whether the reader is willing to accept Chinese literary works, and whether there will be changes in readers' attitudes and feelings under the influence of the work (Lin, 2018). [6] The author believes that Coiling Dragon has achieved a relatively great translation effect from two aspects.

First, it has received a lot of praise and attention abroad. Coiling Dragon ranks among the top ten in the Wuxiaworld fantasy sector all year round, and because of its popularity, it has derived a series of comics, which have been serialized on foreign comic websites such as Manga Raw and have received more than $224 \mathrm{k}$ comments. On the Amazon Goodread website, Coiling Dragon has more than 838 reviews, with a relatively high score of 4.33 points (out of 5 points).

Second, it also allows more overseas users to be willing to contact and understand Chinese culture and give positive feedback to it. Moreover, it inspires overseas readers to create similar novels by imitating Chinese online novels. For instance, Tina Lynge, who is a foreign netizen, has written some online novels by herself because she loves to read Chinese online literature. Blue Phoenix-Sunset City, her most popular online literature work, tells the story of a protagonist named Hui Yue who has gradually become stronger after experiencing betrayal. Besides, there is an original works section on the Gravity Tales website. In this section, original novels by many authors including Tina are published, such as How To Avoid Death On A Daily Basis. GGP, the founder of Gravity Tales, said, 'These novels have imitated Chinese online novels' creative writing methods and publishing ways'. There is also a Chinese learning discussion area in the Wuxiaworld. Some readers want to understand the cultural meaning behind the words "qi", "dao" and "dantian" read in the novel, and others even plan to study Chinese in China because they are anxious that online novels are not updated in time (Lin, 2018). [14]

The successful dissemination of Pan Long overseas reflects the characteristics of the circulation and accumulation of cultural capital. 
According to Bourdieu's Cultural Production Theory, capital, field and habitus can interact and circulate. Different types of capital can be converted through circulation to produce corresponding social effects and results. As a kind of cultural capital, the original work of Pan Long entered the field of translation in a brand-new form after being translated into Coiling Dragon by the translator RWX. In the translation field, the author, the translator, the promotional and publishing platforms have deep interaction and social capital, cultural capital and symbolic capital get accumulated. After being tested by the target language audience, Coiling Dragon has been successfully integrated into the civilization of other countries. Cultural exchanges between nations are conducive to the industrialization of cultural capital. As cultural industries under the situation of overseas online literature market, platforms such as Wuxiaworld and Mango Raw bear the responsibility of translating Pan Long into a cultural product through the membership system and paid channels. Cultural capital and economic capital will produce economic benefits in the successive circular transformation movement, thereby realizing value appreciation.

\section{CONCLUSION}

The author believes that the successful translation and dissemination of Coiling Dragon has closely connected with readers, translators, publishing platforms and promotional platforms. With the accumulation of cultural capital and symbolic capital of the original author and Pan Long, which is seen as a cultural capital with high quality, the translator can make the decision of translating it. The translator has been immersed in American culture since he was young, and is familiar with Chinese culture. Therefore, he has deeply accumulated his cultural capital, which is very helpful for the spreading of Coiling Dragon. The translator has also taken advantage of his social capital, such as the foundation of Wuxiaworld. At the same time, the state has provided adequate policy support for the "going out" of online literature, which has driven the relevant media to carry out positive publicity, so the social capital further accumulated.

The cultural capital and social capital owned by the translator have been continuously accumulated and transformed, which has helped him obtain more symbolic capital. Capital was continuously transformed and expanded like a snowball, and finally converted into economic capital in the market, thereby enhancing the status and qualifications of Coiling Dragon in the field of translation and literature. Coiling Dragon therefore became China's first online literary work successfully exported overseas. When other Chinese online literature is to be translated into foreign languages, it should start from the choice of the original texts, the choice of the translator, the choice of the publishing platform and the choice of promotional tools, and only taking every step patiently can we achieve success.

\section{AUTHORS' CONTRIBUTIONS}

Jialing $\mathrm{Wu}$ is responsible for writing the manuscript, and Chunfang $\mathrm{Yi}$ contributed to revising.

\section{REFERENCES}

[1] (2020). Research report on "Chinese online literature going to sea in 2020". Shanghai iResearch Marketing Consulting Co., Ltd. (eds.) iResearch Series Research Report (pp.500-541).

[2] Xu Tenglong \& Hua Yan. (2020). Looking at the popularity of Chinese online literature from a cross-cultural perspective. Journal of University of Shanghai for Science and Technology (Social Science Edition) (03), 297-300. doi:10.13256/j.cnki.jusst .sse.2020.03.017.

[3] Yuan Yuting, Xia Xiyan \& Chen Qinglan. (2021). The balance of conversion in the English translation of the online novel Coiling Dragon. Overseas English (06), 80-82. doi:CNKI:SUN:HWYY.0.2021-06-036.

[4] Ren Huijun, Wang Jingxuan \& Shi Junhua. (2020). The new translation model of online martial arts novels. Journal of Shangluo University (01), 57-60. doi:10.13440/j.slxy.1674-0033.2020.01.011.

[5] Lv Zhenyan. (2020). Research on the overseas communication of online literature in the context of cross-cultural communication. New Media Studies (17), 78-80. doi:10.16604/j.cnki.issn20960360.2020.17.024.

[6] Lin Ling. (2018) Viewing the Overseas Export of Chinese Network Literature from the 
Theory of Translation and Interlearning Take Coiling Dragon's overseas popularity as an example. Journal of the Three Gorges University (Humanities and Social Sciences Edition) (02), 94-97. doi:10.13393/j.cnki.1672-6219.2018.02.019.

[7] BOURDIEU P, and Wacquant. An Invitation to Reflexive Sociology [M]. Chicago: University of Chicago Press, 1992.

[8] BOURDIEU P. Distinction: a social critique of the judgement of taste [M]. Cambridge: Harvard University Press, 1984.

[9] BOURDIEU P. The forms of capital [C] / / A. H. Halsey, et al. Education: Culture, Economy, and Society. Oxford \& New York: Oxford University Press, 1997: 46-58.

[10] BOURDIEU P. The Field of Cultural Production: Essays on Art and Literature [C]. Cambridge: Polity Press, 1993.

[11] BOURDIEU P. The Specificity of the Scientific Field and the Social Conditions of the Progress of Reason [J]. Social Science Information.1977,14(6):19-47.

[12] Chen Yunuo. (2013). Translation as the construction and circulation of cultural capital (Master's thesis, Hunan University of Science and Technology). https://kns.cnki.net/KCMS/detail/detail.aspx?d bname $=$ CMFD201501 \&filename $=1014022171 . \mathrm{nh}$

[13] Li Yan \& Yang Liu. (2018). Translation of Online Literature: A Path Few Walked-An Interview with Lai Jingping, the Founder of Wuxia World. Translation Forum (04), 3-6. doi:CNKI:SUN:FYLT .0.2018-04-002.

[14] Lin Ling. (2018). Looking at the overseas export of Chinese online literature from the theory of mesotranslatology: Taking the overseas popularity of Coiling Dragon as an example. Journal of China Three Gorges University (Humanities and Social Sciences Edition) (02), 94- 97. doi:10.13393/j.cnki.1672-6219.2018.02.019. 